\title{
Cerebral blood flow imaging in MR-negative SARS-CoV-2-related encephalitis with abnormal psychosis
}

\author{
Kazuma Ota $^{1} \cdot$ Masamune Kimura $^{1}$ (D) $\cdot$ Michi Kawamoto $^{1}$
}

Received: 10 December 2021 / Accepted: 13 February 2022 / Published online: 17 February 2022

(c) The Author(s), under exclusive licence to Springer-Verlag GmbH Germany, part of Springer Nature 2022

SARS-CoV-2 can invade the nervous system and cause neuronal damage, including encephalitis [1]. A 48-yearold man presented with fever and dyspnea (day 0). He was diagnosed with COVID-19 by a positive nasopharyngeal swab of SARS-CoV-2. After the admission, his respiratory condition improved through steroid therapy. He was discharged on day 17. However, he presented to our hospital with an acute severe attention disturbance and excessive masturbation on day 22. His cognitive function was normal and had no paralysis. Blood chemistry analysis revealed no inflammatory response, and a wide screening for antibodies associated with autoimmune encephalitis yielded negative results. The cerebrospinal fluid (CSF) analysis showed a normal cell count; however, the polymerase chain reaction test for SARS-CoV-2 in CSF revealed positive. Brain magnetic resonance imaging (MRI) (day 24, A; fluid-attenuated inversion recovery) revealed no abnormalities, but $\mathrm{N}$-isopropyl$\mathrm{p}-\left[{ }^{123} \mathrm{I}\right]$ iodoamphetamine (IMP) single-photon emission computed tomography (SPECT) (day 27, B; qualitative surface views and $Z$-score views of functional increase by three-dimensional stereotactic surface projection analysis in NEUROSTAT [2]; the whole brain was defined as reference regions for normalization) showed increased uptake in the bilateral thalamus, septal region, and posterior cingulate gyrus. Based on the CSF and the SPECT findings, he was diagnosed with SARS-CoV-2-related encephalitis. Methylprednisolone and intravenous immunoglobulin were started. Following treatment, his psychosis gradually improved, and he fully recovered on day 73 . Repeat ${ }^{123}$ I-IMP SPECT showed improvement in the hyperperfusion areas (C). Some

This article is part of the Topical Collection on Image of the month

\footnotetext{
Masamune Kimura

sumthing1004@gmail.com

1 Department of Neurology, Kobe City Medical Center General Hospital, 2-1-1 Minatojima-minamimachi, Chuo-ku, Kobe, Hyogo 650-0047, Japan
}

cases of SARS-CoV-2-related encephalitis do not show any abnormalities on MRI and require additional imaging [3]. In our case, there was no edematous change on MRI. This might have been because the encephalitis was not too severe to cause edema or early treatment contributed to spare the morphological changes. From a neurological point of view, the thalamus contributes to attention control [4], and septal stimulation elicits a compulsion to masturbate [5]. These localizations were consistent with SPECT findings; thus, the patient's hyperperfusion area might have shown the inflammation with hyperstimulation to the surrounding systems.

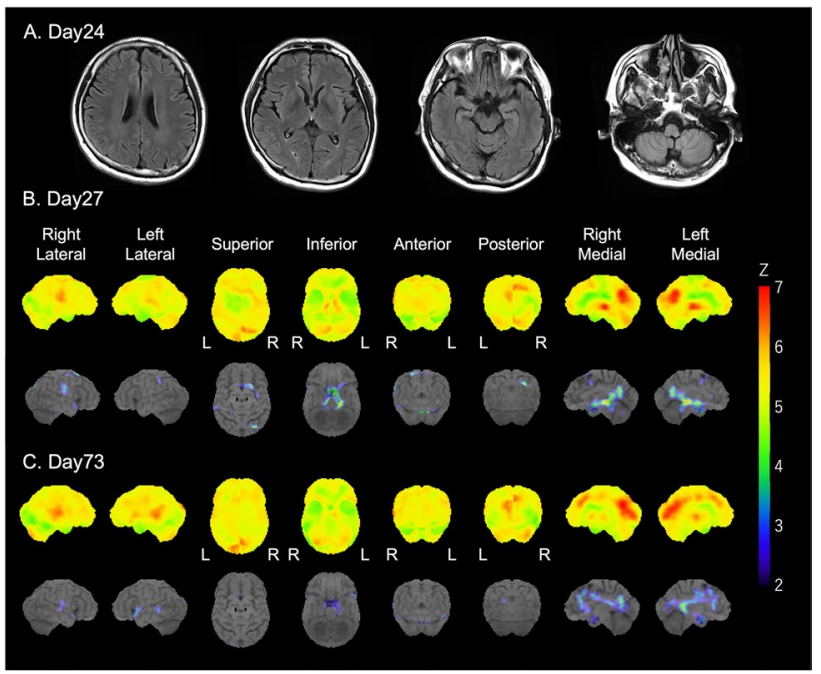




\section{Declarations}

Ethics approval All procedures performed in studies involving human participants were in accordance with the ethical standards of the institutional and/or national research committee and with the 1964 Helsinki declaration and its later amendments or comparable ethical standards.

Consent to participate Informed consent was obtained from all individual participants included in the study.

Conflict of interest The authors declare no competing interests.

\section{References}

1. Wu Y, Xu X, Chen Z, Duan J, Hashimoto K, Yang L, et al. Nervous system involvement after infection with COVID-19 and other coronaviruses. Brain Behav Immun. 2020;87:18-22.
2. Minoshima S, Frey KA, Koeppe RA, Foster NL, Kuhl DE. A diagnostic approach in Alzheimer's disease using three-dimensional stereotactic surface projections of fluorine-18-FDG PET. J Nucl Med. 1995;36:1238-48.

3. Tiraboschi P, Xhani R, Zerbi SM, Corso A, Martinelli I, Fusi L, et al. Postinfectious neurologic complications in COVID-19: a complex case report. J Nucl Med. 2021;62(8):1171-6.

4. Halassa MM, Kastner S. Thalamic functions in distributed cognitive control. Nat Neurosci. 2017;20(12):1669-79.

5. Baird AD, Wilson SJ, Bladin PF, Saling MM, Reutens DC. Neurological control of human sexual behaviour: insights from lesion studies. J Neurol Neurosurg Psych. 2007;78(10):1042-9.

Publisher's note Springer Nature remains neutral with regard to jurisdictional claims in published maps and institutional affiliations. 